\title{
EDITOR'S PAGE
}

\section{Female sexuality after spinal cord lesion}

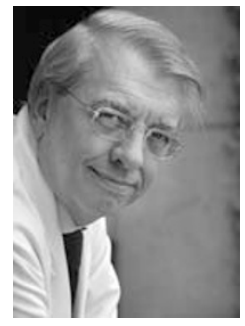

JJ Wyndaele, Editor

Antwerp University Hospital, Antwerp, Belgium

E-mail: spinalcord@uza.be

Dear Spinal Cord reader,

The 49th Annual Scientific meeting of ISCOS in Delhi will live on in the memory as a nice event with good scientific work, nice presentations, productive discussions and useful practical workshops. The Annual General Meeting, at the recommendation of the ISCOS Executive and Council, has reappointed your Editor-in-Chief for another 4 years. The 2009 editorial report will be available soon on the ISCOS website. The next annual meeting, which will be the 50th, will be held in Washington DC, USA-see the ISCOS website for the deadline for the submission of abstracts and workshops. The 50th anniversary of the Society will be highlighted through different initiatives in the Journal.

In this issue there are many important contributions.

As discussed again in Delhi, sexual counseling is an integral part of comprehensive management after spinal cord lesion, and females should not be forgotten. Lombardi et al. carried out a critical review of literature on the multiple aspects of sexual rehabilitation in women with spinal cord injury (SCI) from initial recovery to long-term follow-up. Females with complete tetraplegia deserve special attention immediately at initial recovery as sexual intercourse is much more difficult for them. As more time passes after the injury, patients attain more sexual satisfaction compared to recently injured women. Other topics are also dealt with: neurological changes following SCI and their effect on sexual response; use of sildenafil for female sexuality, lack of detailed analysis on the sexual impact of medical and psychological treatments related to SCI. The authors conclude that sexual issues and response require evaluation during periodic check-ups using validated questionnaires administered by a physician 'guide' who coordinates professional operators, thus providing personalized programmable interventions.

Yuan et al. found, in a rat model, that brain-derived neurotrophin factor has the ability to facilitate the outgrowth of axon in dorsal root ganglia cells, which may have therapeutic potential for SCI. Schültke et al. continued their animal work on quercetin and showed that it attenuates the recruitment of neutrophils to the site of injury. The resulting lower myeloperoxidase release in the injured tissue is likely to decrease the extent of secondary injury and might at least partially explain the neuroprotective effect of the flavonoid quercetin.

Sayenko et al. demonstrated that standing postural control can be improved substantially in both game performance and training-irrelevant tasks in individuals with incomplete SCI using visual feedback.

Bittar and Cliquet Jr demonstrated that the effect of electrical stimulation of the quadriceps and anterior tibial muscles on the feet and ankles of SCI patients was to maintain the feet and ankles in a planted and adequate walking position.

Abel et al. showed that ultra-long term races $(540 \mathrm{~km})$ in a handcycle can be properly undertaken by persons with a SCI, but that the quality of the training preparation time is of immense importance for reaching adequate physiological capacity and for avoiding serious injuries or medical problems.

Rangappa et al., in a retrospective medical record review, showed that patients with a cervical SCI needing a cardiac pacemaker are characterized by a higher level of SCI injury and motor loss, require mechanical respiratory and inotropic support, a tracheostomy to wean, and bradycardic episodes of a later onset and over a longer period of time. They should be managed at hospitals with specialized acute spinal injury, intensive care and cardiac pacemaker services.

García-Leoni et al. found a significant risk of pneumonia in 100 long term ventilator-assisted individuals, but low mortality with proper treatment.

Yasmeen et al. found a high incidence of faecal incontinence in individuals with chronic SCI in Pakistan despite the use of suppositories, laxatives and bulk forming agents. Adherence to a standard bowel care programme and practices are influenced by sociocultural factors and a lack of education on the part of the patient.

Cosar et al. found in a retrospective, 12-year case series that, although patients with non-traumatic SCI had shorter lengths of stay and higher ASIA scores, there was no significant difference in functional outcomes with traumatic injuries.

ISCOS REQUESTS ALL MEMBERS TO FORWARD THEIR EMAIL ADDRESS TO admin@iscos.org.uk, BEFORE THE END OF THE YEAR AS COMMUNICATION BETWEEN THE CENTRAL OFFICE AND MEMBERS WILL BE MADE BY EMAIL ALONE IN FUTURE. 